\title{
Atomic Fermi-Bose Mixtures in Inhomogeneous and Random Lattices: From Fermi Glass to Quantum Spin Glass and Quantum Percolation
}

\author{
A. Sanpera ${ }^{1,2}$, A. Kantian ${ }^{2}$, L. Sanchez-Palencia ${ }^{2}$, J. Zakrzewski ${ }^{3}$, and M. Lewenstein ${ }^{1,2}$ \\ ${ }^{1}$ Institut de Ciències Fotòniques, 08034 Barcelona, Spain \\ ${ }^{2}$ Institut für Theoretische Physik, Universität Hannover, D-30167 Hannover, Germany \\ ${ }^{3}$ Instytut Fizyki, Uniwersytet Jagielloński, PL-30-059 Kraków, Poland
}

(Dated: 1st November 2018)

\begin{abstract}
We investigate atomic Fermi-Bose mixtures in inhomogeneous and random optical lattices in the limit of strong atom-atom interactions. We derive the effective Hamiltonian describing the dynamics of the system and discuss its low temperature physics. We demonstrate possibility of controlling the interactions at local level in inhomogeneous but regular lattices. Such a control leads to the achievement of Fermi glass, quantum Fermi spin glass, and quantum percolation regimes involving bare and/or composite fermions in random lattices.
\end{abstract}

PACS numbers: 03.75.Kk,03.75.Lm,05.30.Jp,64.60.Cn

Fermi-Bose (FB) mixtures attract considerable interest in the physics of ultra-cold atomic and molecular gases, comparable with the interest in molecular BoseEinstein condensation (BEC) 1], or Bardeen-CooperSchrieffer (BCS) transition 2] in ultra-cold Fermi mixtures. The reason for interest in FB systems is threefold. First, these are very fundamental systems that have no direct analogues in condensed matter physics. Second, these systems can be very efficiently cooled using sympathetic cooling down to very low temperatures $T$ (of order of tens $\mathrm{nK}$ ) 3, 4, 5, 6]. Finally, their physics is extremely rich and not yet fully understood.

FB mixtures have been intensively studied in traps [7], but the experimental observation of the superfluid (SF) to Mott-insulator (MI) transition in bosonic gases 14], predicted in Ref. [15], has triggered the interest in the physics of FB mixtures in optical lattices [16]. Under appropriate conditions such mixtures can be well described by the Fermi-Bose Hubbard model (FBH) 17]. A particularly appealing feature of the FBH model is the possibility to produce novel quantum phases [18], fermion-boson induced superfluidity [19], and composite fermions, which for attractive (repulsive) interactions between fermions and bosons, are formed by a fermion and bosons (bosonic holes) as first shown in [20] (see also [21, 22]).

FB mixtures in the limit of strong atom-atom interactions (strong coupling regime) show a very rich variety of quantum phases in periodic optical lattices 21]. They include the mentioned composite fermions, and range from a normal Fermi liquid, a density wave, a superfluid liquid, to an insulator with fermionic domains. The phase diagram of the system has been determined in Ref. 23] by means of mean-field theory [24]. These studies have been generalized recently to inhomogeneous lattices 25 . to include the effects of the lattice and of a possible trap potential. So far, only the case of strong interactions and vanishing hopping has been considered.

In the present Letter we study the low temperature physics of FB mixtures in optical lattices with local and random inhomogeneities in the strong interactions limit but including tunneling as a perturbation. We show that interactions and tunneling may be controlled at the local level in inhomogeneous lattices [26]. This control gives access to a wide variety of regimes and we derive the corresponding effective Hamiltonians. We then show how to achieve Fermi glass, fermionic spin glass and quantum percolation regimes involving bare and/or composite fermions in random lattices.

We consider a sample of ultra-cold bosonic and (polarized) fermionic atoms (e.g. ${ }^{7} \mathrm{Li}^{6}{ }^{6} \mathrm{Li}$ or ${ }^{87} \mathrm{Rb}^{-}{ }^{40} \mathrm{~K}$ ) trapped in an optical lattice. At low temperature, the atoms occupy only the lowest energy band and it is convenient to work in the corresponding Wannier basis 15. Note that a fermion number $N_{F}$ strictly smaller than the number of lattice sites $N$ is required here. The Hamiltonian of the system reads [17, 27]:

$$
\begin{aligned}
& H_{\mathrm{FBH}}=-\sum_{\langle i j\rangle}\left(J_{\mathrm{B}} b_{i}^{\dagger} b_{j}+J_{\mathrm{F}} f_{i}^{\dagger} f_{j}+\text { h.c. }\right) \\
& +\sum_{i}\left[\frac{1}{2} V n_{i}\left(n_{i}-1\right)+U n_{i} m_{i}-\mu_{i}^{B} n_{i}-\mu_{i}^{F} m_{i}\right],
\end{aligned}
$$

where $b_{i}^{\dagger}, b_{j}, f_{i}^{\dagger}, f_{j}$ are the bosonic and fermionic creation-annihilation operators, $n_{i}=b_{i}^{\dagger} b_{i}$ and $m_{i}=f_{i}^{\dagger} f_{i}$. The FBH model describes: i) nearest neighbor (n.n.) boson (fermion) hopping, with an associated negative energy, $-J_{\mathrm{B}}\left(-J_{\mathrm{F}}\right)$; ii) on-site repulsive boson-boson interactions with an energy $V$; iii) on-site boson-fermion interactions with an energy $U$, which is positive (negative) for repulsive (attractive) interactions, iv) and, finally, interactions with the optical potential, with energies $\mu_{i}^{\mathrm{B}}$ and $\mu_{i}^{\mathrm{F}}$. In the following, we shall consider only the case $J_{\mathrm{B}}=J_{\mathrm{F}}=J$ and the regime of strong interactions, namely $V, U \gg J$.

In a periodic optical lattice, $\mu_{i}^{\mathrm{B}, \mathrm{F}}$ is simply the (bosons or fermions) chemical potential and is independent of the site $i$. It is, however, possible to add a laser field independent of the lattice to modify the depths of the optical 
potential wells in a site-dependent way [28]. In this case, the local potential depth has to be added to $\mu_{i}^{\mathrm{B}, \mathrm{F}}$, which may now be inhomogeneous. If the added field is periodic and if the spatial period is commensurate with the lattice period, $\mu_{i}^{\mathrm{B}, \mathrm{F}}$ is periodic; if the spatial periods are incommensurate, $\mu_{i}^{\mathrm{B}, \mathrm{F}}$ is quasi-periodic. One can also add a random speckle field, so that $\mu_{i}^{\mathrm{B}, \mathrm{F}}$ is random. Experimental techniques offer full possibilities to control such periodic, quasi-periodic, or disordered $\mu_{i}^{\mathrm{B}, \mathrm{F}}$ [29]. Note, that the additional inhomogeneous potential might, but does not have to, act equally on both atomic species. Here, we will study the case $\mu_{i}^{F}=0, \mu_{i}^{B}=\mu_{i} V$.

In Ref. 21] we have used the method of degenerate second order perturbation theory to derive an effective Hamiltonian by projecting the wave function onto the multiply degenerate ground state of the system in the absence of tunneling. This can be extended to the present situation, where there are very many states with similar energies. It is thus reasonable to project the wave function on the manifold of 'ground states'. These states are local minima of energy, since at least some of hopping acts increase their energy by $V$ or $|U|$.

Let us consider first $J=0$, and the case $0 \leq \mu_{i}<1$. In the absence of a fermion one expects one boson per site, i.e. $n_{i}=1$ [30]. We shall consider here only the case of repulsive interactions, i.e. $\alpha=U / V>0$.

It is useful to divide the sites into : i) $A$-sites, for which $\mu_{i}-\alpha \geq 0$, and fermions do not push bosons out, and ii) $B$-sites, for which $\mu_{i}-\alpha<0$, and the fermion pushes the boson out forming a composite fermion-bosonic hole. Energetically, the second situation is favorable, so for a given set of $N^{\mathrm{A}}$ of $A$-, and $N^{\mathrm{B}}=N-N^{\mathrm{A}}$ of $B$-sites, the fermions at very low temperature will first occupy the $B$-sites until $N_{\mathrm{F}}=N^{\mathrm{B}}$, and then they will start to occupy the $A$-sites. We construct the corresponding projector operators $P, Q=1-P$, which depend on $N^{\mathrm{A}}$ and $N_{\mathrm{F}}$. The operator $P$ describes the projection onto the manifold of quasi-degenerated states in which the fermions occupy the $B$-sites stripped of bosons and some $A$-sites only if $N_{\mathrm{F}} \geq N^{\mathrm{B}}$. In this case there is a boson in any $A$-site and if $N_{\mathrm{F}}<N^{\mathrm{B}}$ there is also a boson in the $B$-sites which do not contain fermions. We use the formalism of second order time dependent perturbation theory [32], and project the Schrödinger equation, $i \hbar \partial_{t}|\psi(t)\rangle=\left(H_{0}+H_{1}\right)|\psi(t)\rangle$, onto the manifold of states spanning $P$. The "zeroth-order" part $H_{0}$ contains the atomic interactions and terms proportional to the chemical potential and commutes with $P . H_{1}$ represents the tunneling terms. The effective equation for $\left|\psi_{P}\right\rangle=P|\psi\rangle$ reads then $i \hbar \partial_{t}\left|\psi_{P}(t)\right\rangle=H_{\text {eff }}\left|\psi_{P}(t)\right\rangle$ where

$$
\begin{aligned}
& \left\langle\text { out }\left|H_{\mathrm{eff}}\right| i n\right\rangle=\left\langle\text { out }\left|H_{0}\right| \text { in }\right\rangle+\left\langle\text { out }\left|P H_{1} P\right| \text { in }\right\rangle \\
& -\frac{1}{2}\left\langle\text { out }\left|P H_{1} Q\left(\frac{1}{H_{0}-E_{\text {in }}}+\frac{1}{H_{0}-E_{\text {out }}}\right) Q H_{1} P\right| \text { in }\right\rangle .
\end{aligned}
$$

At low temperature the physics of the system depends
on the relation between $\mu_{i}$ 's and $\alpha$. For small inhomo-
geneities, we may neglect the contributions of $\Delta_{i j}$ to

At low temperature the physics of the system depends
on the relation between $\mu_{i}$ 's and $\alpha$. For small inhomo-
geneities, we may neglect the contributions of $\Delta_{i j}$ to

At low temperature the physics of the system depends
on the relation between $\mu_{i}$ 's and $\alpha$. For small inhomo-
geneities, we may neglect the contributions of $\Delta_{i j}$ to

The effective Hamiltonian $H_{\text {eff }}$ has the form

$$
H_{\mathrm{eff}}=\sum_{\langle i j\rangle}\left[-\left(d_{i j} F_{i}^{\dagger} F_{j}+\text { h.c. }\right)+K_{i j} M_{i} M_{j}\right]+\sum_{i} \tilde{\mu}_{i} M_{i},
$$

where $F_{i}, F_{i}^{\dagger}$ are the corresponding (composite) fermionic annihilation and creation operators, and $M_{i}=F_{i}^{\dagger} F_{i}$. The hopping amplitudes $d_{i j}$ and the n.n. couplings $K_{i j}$ (which might be repulsive $(>0)$ or attractive $(<0)$ ) are of order of $J^{2} / V$. The values of the couplings depend on $\alpha, \tilde{\mu}_{i}, \tilde{\mu}_{j}$, and $J$, and have to be determined carefully for different cases, as discussed below. Note, however, that the hopping $i \rightarrow j$, or back causes the energy change $\pm\left(\Delta_{i j}=\mu_{i}-\mu_{j}\right)$ in units of $V$, i.e is highly nonresonant and inefficient for $\Delta_{i j} \simeq 1$; it will first lead to jump rates of order $O\left(J^{4} / V^{3}\right)$. Additionally, composite fermions may feel the local energy $\tilde{\mu}_{i}$.

I) All sites are of type B. In this case we have a gas of composites flowing within the MI with 1 boson per site. The couplings are

$$
\begin{aligned}
d_{i j} & =\frac{J^{2}}{V}\left(\frac{\alpha}{\alpha^{2}-\left(\Delta_{i j}\right)^{2}}+\frac{1}{\alpha}\right) \\
K_{i j} & =-\frac{J^{2}}{V}\left(\frac{4}{1-\left(\Delta_{i j}\right)^{2}}-\frac{2}{\alpha}-\frac{2 \alpha}{\alpha^{2}-\left(\Delta_{i j}\right)^{2}}\right) .
\end{aligned}
$$

The chemical potential $\tilde{\mu}_{i} / V \simeq \mu_{i}$ up to corrections of order $O\left(J^{2} / V\right)$. The hopping amplitudes $d_{i j}$ are for this case always positive, although may vary quite significantly with disorder, especially when $\Delta_{i j} \simeq \alpha$. As shown in Fig. 1, for $\alpha>1, K_{i j} \leq 0$ and we deal with attractive (although random) interactions. For $\alpha<1$, but close to $1, K_{i j}$ might take positive or negative values for $\Delta_{i j}$ small or $\Delta_{i j} \simeq \alpha$. In this case the qualitative character of interactions is controlled by inhomogeneity.



Figure 1: Nearest neighbor couplings $K_{i j}$ as a function of $\Delta_{i j}$. Solid line: Coupling in case I, with $\alpha_{I}=0.93$. Dashed line: Same expression with $\alpha_{I}=1.07$. Dashed dotted line: Coupling in case II with $\alpha_{I I}=0.03$. 
$d_{i j} \simeq d$ and $K_{i j} \simeq K$, and keep only the leading disorder contribution in $\tilde{\mu}_{i}$. Note, that the latter contribution is relevant in $1 \mathrm{D}$ and 2D leading to Anderson localization of single particles 33 . When $K \ll d$ the system will then be in the Fermi glass phase, i.e. Anderson localized (and many-body corrected) single particle states will be occupied according to the Fermi-Dirac rules 34 . For repulsive interactions and $K \gg d$, the ground state will be a Mott insulator for large enough filling factors. In particular, for filling factor $1 / 2$ one expects to form a checker-board phase. For intermediate values of $K / d$ delocalized metallic phases with enhanced persistent currents are possible 35. Similarly, for attractive interactions $(K<0)$ and $|K|<d$ one expects competition between pairing of fermions and disorder. In this case for $|K| \gg d$, the fermions will form a domain insulator.

Another interesting limit is when $\left|\Delta_{i j}\right| \simeq \alpha \simeq 1$. The tunneling becomes then non-resonant and negligible, while the couplings $K_{i j}$ fluctuate strongly. We end up then with the (fermionic) Ising spin glass model 36] described by the Edwards-Anderson model [37]:

$$
H_{\mathrm{E}-\mathrm{A}}=\frac{1}{4} \sum_{\langle i j\rangle} K_{i j} s_{i} s_{j}+\sum_{i} \tilde{\mu}_{i} s_{i} / 2
$$

with $s_{i}=2 M_{i}-1= \pm 1$. The above Hamiltonian is well approximated by a random one with Gaussian and independent distributions for $K_{i j} / 4$ and $\tilde{\mu}_{i} / 2$ with mean $0(H)$, and variances $K(h)$, respectively. In this limit the system may be used to study various open questions of spin glass physics, concerning the nature of ordering (Parisi's [37] versus "droplet" picture [38, 39]), broken symmetry and dynamics in classical (in absence of hopping) and quantum (with small, but nevertheless present hopping) spin glasses [24, 40]. The predictions of Parisi's mean field theory for the model (6) can be obtained by replacing the model by the corresponding SherringtonKirkpatrick model, and employing the standard method of replica trick [37]. The calculations differ from the standard ones in that the constraint of fixed mean number of fermions is applied, and one deals simultaneously with random couplings and "magnetic fields" $\tilde{\mu}_{i}$. Following de Almeida and Thouless (A-T) approach [41], we obtain the A-T surface separating the stable paramagnetic state from the "true" spin glass state, characterized by replica symmetry breaking, and ultrametrically arranged ground states. The paramagnetic state is stable for

$$
\left(\frac{k_{\mathrm{B}} T}{K}\right)^{2}>\left\langle\left\langle\operatorname{sech}^{4}\left(\frac{x \sqrt{K^{2} q+h^{2}}+H}{k_{\mathrm{B}} T}\right)\right\rangle\right\rangle_{x},
$$

where $q=\left\langle\left\langle\tanh ^{2}\left(\frac{x \sqrt{K^{2} q+h^{2}}+H}{k_{\mathrm{B}} T}\right)\right\rangle\right\rangle_{x}$, the constraint is $m=\left\langle\left\langle\tanh \left(\frac{x \sqrt{K^{2} q+h^{2}}+H}{k_{\mathrm{B}} T}\right)\right\rangle\right\rangle_{x}^{x}$, with $m=$ $2 N_{\mathrm{F}} / N-1$ and $\langle\langle.\rangle\rangle_{x}$ denotes averaging over normally distributed random variable $x$ which represents disorder within the replica method 37 . Note, that according to the predictions of the alternative "droplet" model [38], applied to (6), no A-T surface is expected to exist.

II) All sites are of type $A$. In this case $\alpha<1$, and we have a gas of bare fermions flowing over the MI with 1 boson per site. The coefficients are

$$
\begin{aligned}
d_{i j} & =J, \quad K_{i j}=-\frac{J^{2}}{V}\left(\frac{8}{1-\left(\Delta_{i j}\right)^{2}}\right. \\
& \left.-\frac{4(1+\alpha)}{(1+\alpha)^{2}-\left(\Delta_{i j}\right)^{2}}-\frac{4(1-\alpha)}{(1-\alpha)^{2}-\left(\Delta_{i j}\right)^{2}}\right),
\end{aligned}
$$

and $\tilde{\mu}_{i} \simeq 0$ up to corrections of order $O\left(J^{2} / V\right)$. The couplings $K_{i j}$ are positive, and for $\alpha \simeq 0, K_{i j} \simeq O\left(\alpha^{2}\right)$, and both the repulsive interactions, and disorder are very weak, leading to a Fermi liquid behavior at low $T$. For finite $\alpha$, and $\Delta_{i j} \simeq 1-\alpha$, however, the fluctuations of $K_{i j}$ might be quite large. Note, that for $\alpha \simeq 1$, this will occur even for small disorder. Assuming for simplicity that $K_{i j}$ take either very large, or zero value, we see that the physics of bond percolation [42] will play a role. The bonds will form a "weak" and "strong" clusters, each of which may be percolating. The fermions will hope freely in the "weak" cluster; only one fermion per bond will be allowed in the "strong" cluster.

III) Both $N^{A}$ and $N^{B}$ of order $N / 2$. In this case the physics of site percolation 42 will be relevant. If $N_{\mathrm{F}} \leq N^{\mathrm{B}}$ the composite fermions will move within a cluster of $B$ sites. When $N^{\mathrm{B}}$ is above the classical percolation threshold, this cluster will be percolating. The expressions Eq. (4) and Eq. (5) will still be valid, except that they will connect only the $B$-sites.

The physics of the system will be similar as in the case I), but it will occur now on the percolating cluster. For small disorder, and $K \ll d$ the system will be in a Fermi glass phase in which the interplay between the Anderson localization of single particles due to fluctuations of $\mu_{i}$ and quantum percolation effects, that is randomness of the $B$-sites cluster, will occur. For repulsive interactions and $K \gg d$, the ground state will be a Mott insulator on the cluster for large filling factors. It is an open question whether the delocalized metallic phases with enhanced persistent current of the kind discussed in Ref. 35 might exist in this case. Similarly, it is an open question whether for attractive interactions $(K<0)$ and $|K|<d$ pairing of (perhaps localized) fermions will take place. If $|K| \gg d$, we expect the fermions to form a domain insulator on the cluster.

In the "spin-glass" limit $\Delta_{i j} \simeq \alpha \simeq 1$, we deal with the Edwards-Anderson spin glass on the cluster. Such systems are of interest in condensed matter physics [43], and again questions connected to the nature of spin glass ordering may be studied in this case.

When $N_{\mathrm{F}}>N^{\mathrm{B}}$, all $B$-sites will be filled, and the physics will occur on the cluster of $A$ sites. For $\alpha \simeq 0$, 
we shall deal with a gas with very weak repulsive interactions, and no significant disorder on the random cluster. This is an ideal test ground to study quantum percolation at low T. For finite $\alpha$, and $\Delta_{i j} \simeq 1-\alpha$, the interplay between the fluctuating repulsive $K_{i j}$ 's and quantum percolation might be studied.

Summarizing, we have studied atomic Fermi-Bose mixtures in optical lattices in the strong interaction limit, and in the presence of an inhomogeneous, or random onsite potential. We have derived the effective Hamiltonian describing the low temperature physics of the system, and shown that an inhomogeneous potential may be efficiently used to control the nature and strength of (boson mediated) interactions in the system. Using a random potential, one is able to control the system in such a way that its physics corresponds to a whole variety of quantum disordered systems: Fermi glass, fermionic spin glass, and quantum percolation systems.

We thank M. Baranov, H.-P. Büchler, A. Georges, J. Wehr, J. Parrondo and P. Zoller for fruitful discussions. We acknowledge support from the Deutsche Forschungsgemeinschaft (SFB 407 and SPP1116), the RTN Cold Quantum Gases, ESF PESC BEC2000+, the Alexander von Humboldt Foundation and KBN grant PBZ-MIN008/P03/2003 (J.Z.).

[1] S. Jochin et al., Science Express 302, 2101 (2003); M. Greiner, C.A. Regal, and D.S. Jin, Nature 426, 537 (2003); M. W. Zwierlein et al., Phys. Rev. Lett. 91, 250401 (2003).

[2] G. V. Shlyapnikov, Proc. XVIII Int. Conf. Atom. Phys., Eds. H.R- Sadeghpour, D.E. Pritchard, and E.J. Heller (World Scientific, Singapore, 2002).

[3] A. G. Truscott et al., Science 291, 2570 (2001).

[4] F. Schreck et al., Phys. Rev. Lett. 87, 080403 (2001).

[5] Z. Hadzibabic et al., Phys. Rev. Lett. 88, 160401 (2002); Z. Hadzibabic et al., cond-mat/0306050

[6] G. Modugno et al., Science 297, 2240 (2002).

[7] The studies of trapped FB gases concerned in particular FB phase separation [8, 9], the phase diagram [10], novel types of collective modes 9, 11], Fermi-Fermi interactions mediated by bosons [9, 12], the collapse of the Fermi cloud in the presence of attractive FB interactions [6], or the effects characteristic for the 1D FB mixtures [13].

[8] K. Mølmer, Phys. Rev. Lett. 80, 1804-1807 (1998).

[9] M.J. Bijlsma, B.A. Heringa, and H.T.C. Stoof, Phys. Rev. A 61, 053601 (2000); H. Pu, W. Zhang, M. Wilkens, and P. Meystre, Phys. Rev. Lett. 88, 070408 (2002).

[10] R. Roth, Phys. Rev. A 66, 013614 (2002).

[11] P. Capuzzi and E. S. Hernández, Phys. Rev. A 64, 043607 (2001); X.-J. Liu and H. Hu, ibid. 67, 023613 (2003).

[12] A. Albus et al., Phys. Rev. A 65, 053607 (2002); L. Viverit, and S. Giorgini, ibid. 66, 063604 (2002); H. Heiselberg, C. J. Pethick, H. Smith and L. Viverit, Phys. Rev. Lett. 85, 2418 (2000).

[13] K. K. Das, Phys. Rev. Lett. 90, 170403 (2003); M. A. Cazalilla and A. F. Ho, ibid. 91, 150403 (2003).
[14] M. Greiner et al., Nature 415, 39 (2002).

[15] D. Jaksch et al., Phys. Rev. Lett. 81, 3108 (1998).

[16] G. Modugno et al., Phys. Rev. A 68, 011601(R) (2003).

[17] A. Albus, F. Illuminati, and J. Eisert, Phys. Rev. A 68, 023606 (2003).

[18] H. P. Büchler and G. Blatter, Phys. Rev. Lett. 91, 130404 (2003); R. Roth and K. Burnett, Phys. Rev. A 68, 023604 (2003).

[19] F. Illuminati and A. Albus, cond-mat/0311248

[20] A. B. Kuklov and B. V. Svistunov, Phys. Rev. Lett. 90, 100401 (2003).

[21] M. Lewenstein, L. Santos, M. A. Baranov and H. Fehrmann, Phys. Rev. Lett. 92, 050401 (2004).

[22] This phenomenon, related to the appearance of counterflow superfluidity in Ref. [20], may occur also in the absence of the optical lattice, M. Yu. Kagan, D. V. Efremov, and A.V. Klaptsov, cond-mat/0209481

[23] H. Fehrmann et al., cond-mat/0307635

[24] S. Sachdev, Quantum Phase Transitions, (Cambridge University Press, Cambridge, 1999).

[25] M. Cramer, J. Eisert, and F. Illuminati, cond-mat/0310705

[26] For control in other context, see L.-M. Duan, E. Demler and M. D. Lukin, Phys. Rev. Lett. 91090402 (2003).

[27] A. Auerbach, Interacting Electrons and Quantum magnetism, (Springer, New York, 1994).

[28] B. Damski et al., Phys. Rev. Lett. 91, 080403 (2003).

[29] L. Guidoni and P. Verkerk, Phys. Rev. A 57, R1501 (1999); L. Guidoni, C. Triché, P. Verkerk, and G. Grynberg, Phys. Rev. Lett. 79, 3363 (1997); P. Horak, J.-Y. Courtois, and G. Grynberg, Phys. Rev. A 58, 3953-3962 (1998).

[30] In current experiments [14], the exact commensurate filling factor 1 appears in the central region of the lattice due to the presence of a weak confining potential [31].

[31] G. Batrouni et al., Phys. Rev. Lett. 89, 117203 (2002).

[32] C. Cohen-Tannoudji, J. Dupont-Roc, G. Grynberg, Atom-photon interactions: basic Processes and Applications (Wiley, New York, 1992).

[33] E. Abrahams, P. W. Anderson, D. C. Licciardello and T. V. Ramakrishnan, Phys. Rev. Lett. 42, 673 (1979).

[34] R. Freedman and J. A. Hertz, Phys. Rev. B 15, 2384 (1977).

[35] P. Schmitteckert, R. A. Jalabert, D. Weinmann and J.-L. Pichard, Phys. Rev. Lett. 81, 2308 (1998); G. Benenti, X. Waintal, and J.-L. Pichard, ibid. 83, 1826 (1999); E. Gambeti-Césare et al., Europhys. Lett. 60, 120 (2002).

[36] R. Oppermann and B. Rosenow, Phys. Rev. B 60, 10325 (1999); R. Oppermann and D. Sherrington, ibid. 67, 245111 (2003).

[37] M. Mézard, G. Parisi, and M.A. Virasoro, Spin Glass and Beyond (World Scientific, Singapour, 1987).

[38] D. S. Fisher and D. A. Huse, Phys. Rev. Lett. 56, 1601 (1986); A. J. Bray and M. A. Moore, ibid. 58, 57 (1987).

[39] C. M. Newman and D. L. Stein, J. Phys.-Condens. Mat. 15, R1319 (2003).

[40] A. Georges, O. Parcollet, and S. Sachdev, Phys. Rev. B 63, 134406 (2001).

[41] J. R. L. de Almeida and D. J. Thouless, J. Phys. A 11, 983 (1978).

[42] A. Aharony and D. Stauffer, Introduction to percolation Theory (Taylor \& Francis, London, 1994).

[43] P. H. R. Barbosa, E. P. Raposo, and M. D. CoutinhoFilho, Phys. Rev. Lett. 91, 197207 (2003). 